\title{
Identification of syrup type using fourier transform-near infrared spectroscopy with multivariate classification methods
}

\author{
Ravipat Lapcharoensuk* and Natrapee Nakawajana \\ Department of Agricultural Engineering \\ Faculty of Engineering \\ King Mongkut's Institute of Technology Ladkrabang \\ Bangkok 10520, Thailand \\ *ravipat.la@kmitl.ac.th
}

Received 2 March 2017

Accepted 29 June 2017

Published 20 July 2017

\begin{abstract}
This research aimed to establish near infrared (NIR) spectroscopy models for identification of syrup types in which the maple syrup was discriminated from other syrup types. Thirty syrup types were used in this research; the NIR spectra of each type were recorded with 10 replicates. The repeatability and reproducibility of NIR scanning were performed, and the absorbance at $6940 \mathrm{~cm}^{-1}$ was used for calculation. Principal component analysis was used to group the syrup type. Identification models were developed by soft independent modeling by class analogy (SIMCA) and partial least-squares discriminant analysis (PLS-DA). The SIMCA models of all syrup types exhibited accuracy percentage of $93.3-100 \%$ for identifying syrup types, whereas maple syrup discrimination models showed percentage of accuracy between $83.2 \%$ and $100 \%$. The PLS-DA technique gave the accuracy of syrup types classification between $96.6 \%$ and $100 \%$ and presented ability on discrimination of maple syrup form other types of syrup with accuracy of $100 \%$. The finding presented the potential of NIR spectroscopy for the syrup type identification.
\end{abstract}

Keywords: Identification; NIR spectroscopy; syrup; multivariate classification.

\section{Introduction}

Syrup is a sweet flavoring that is a viscous liquid and comprises a concentrated solution of sucrose and other sugars in water. The viscosity of syrup arises from the multiple hydrogen bonds between the dissolved sugars, which has many hydroxyl $(\mathrm{OH})$ group and the water. Many people eat syrup on waffles or pancakes and sometimes it is used to make cakes, cookies and other desserts. Syrup can be produced from dissolving sugar in the water or

*Corresponding author.

This is an Open Access article published by World Scientific Publishing Company. It is distributed under the terms of the Creative Commons Attribution 4.0 (CC-BY) License. Further distribution of this work is permitted, provided the original work is properly cited. 
made from a naturally sweet juice such as corn syrup, cane syrup, agave syrup, maple syrup, etc.

Maple syrup is obtained from the sap of sugar maple trees (Acer saccharum Marsh). Sap is a liquid inside sugar maple trees; approximately $2-6 \%$ of the sap is sucrose. Maple syrup is made by simmering the sap until it is viscous and then filter out the sediment, the remaining solution is maple syrup. The chemical composition of maple syrup is dominated by carbohydrates, mainly more than $80 \%$ of sucrose, followed by variable detectable traces of glucose and fructose, minerals, vitamins and organic acids. ${ }^{1-4}$

Near-infrared (NIRs) spectroscopy technique has been used extensively to investigate the parameters in syrup. Luqing et al. ${ }^{5}$ reported that using NIRs for detection and quantification of sugar and glucose syrup in roasted green tea, the results showed that NIR has a good potential in quantifying sugar and glucose syrup content in roasted green tea and for detecting adulterated roasted green tea sample. The NIRs have been used to detect adulteration of Irish honey by beet invert syrup and high fructose corn syrup in transflectance mode by Kelly et al. ${ }^{6}$ The result has demonstrated that NIRs could be used as a rapid screening tool for the detection of beet invert syrup and high fructose corn syrup adulteration in Irish honey at a minimum concentration, which may be commercially useful. Furthermore, NIRs have been used for qualitative and quantitative detection of honey adulterated with high-fructose corn syrup and maltose syrup, and the results showed that NIRs have a potential as a method for the classification of authentic and adulterated honeys. ${ }^{7}$

Two classification methods, which are soft independent modeling of class analogy (SIMCA) and partial least-squares discriminant analysis (PLS-DA), have been used to build classification models for discrimination of agricultural products. Veleva-Doneva et al. ${ }^{8}$ reported that using SIMCA to build the models for detection of bacterial contamination in milk using NIRS, ${ }^{8}$ using PLS-DA to establish the classification models to discriminate the oat and groat kernels by Serranti et al. ${ }^{9}$ developing PLS-DA models for prediction of diazinon contents in the samples and for classification of intact cucumbers, ${ }^{10}$ SIMCA and PLS-DA were used to build the models for nondestructive detection of blackspot in potatoes. ${ }^{11}$
From the aforementioned reports, the NIRs can be potentially used to investigate the parameters of syrup in fast, nonsample preparation and nondestructive fashion. Hence, the research aims to establish NIR models for identification of syrup types and discrimination of maple syrup from other syrup types; the resulting data of NIRs are analyzed applying principal component analysis (PCA) to transform the original data to a new group and PLS-DA and SIMCA to build the classification models to identify and discriminate the syrups.

\section{Materials and Methods}

\subsection{Syrup sample}

A total of 30 syrup samples from different sources were collected from supermarkets and convenient stores as outlined below: five samples of maple syrup (MPS1, MPS2, MPS3 MPS4 and MPS5), four samples of honey (HN1, HN2, HN3 and HN4), three samples of cane syrup (CNS1, CNS2 and CNS3), three samples of hazelnut syrup (HZS1, HZS2 and HZS3), two samples of maple-flavored syrup (MFS1 and MFS2), two samples of corn syrup (COS1 and COS2), two samples of caramel syrup (CMS1 and CMS2), two samples of vanilla-flavored syrup (VFS1 and VFS2), two samples of mint-flavored syrup (MIS1 and MIS2), one sample of butterscotch syrup (BSS), one sample of blue-flavored syrup (BFS), one sample of carob syrup (CRS), one sample of agave syrup (AGS) and one sample of coconut flower syrup (CFS). Syrup samples were delivered to Laboratory of NIRS Research Center for Agricultural Product and Food, Curriculum of Agricultural Engineering, Department of Mechanical Engineering, Faculty of Engineering, King Mongkut's Institute of Technology Ladkrabang, Bangkok, Thailand. Immediately prior to the experiment, the samples were kept at room temperature $\left(25^{\circ} \mathrm{C}\right)$ for $30 \mathrm{~min}$ before NIR scanning.

\subsection{Sample scanning}

Each syrup sample was poured into a vial $(20 \mathrm{~mm}$ diameter and $43 \mathrm{~mm}$ height), and the syrup sample was pressed by the transmittance probe (path length $0.35 \mathrm{~mm}$ ). NIR spectra of syrup samples were recorded in reflection mode between $12,500 \mathrm{~cm}^{-1}$ and $4000 \mathrm{~cm}^{-1}(800-2500 \mathrm{~nm})$ at a resolution of $8 \mathrm{~cm}^{-1}$ by the Fourier transform (FT)-NIR 
spectrometer (MPA, Bruker Ltd, Germany). Ten spectra were collected from each syrup sample. Figure 1 shows syrup spectra measurement by FTNIR spectrometer.

\subsection{NIR identification models established and data analysis}

The identification models were created for two cases: identified type of syrup and discriminated maple syrup from other syrup types. The 300 spectra of syrup samples were used to create identification models. Identification models were performed with the multivariate statistical program of Unscrambler v10.1 software package (CAMO AS, Trondheim, Norway). Principle component analysis (PCA) was used for grouping the syrup type. The error of the PCA model was then calculated with the full cross validation method. Identification models were established using soft independent modeling by class analogy (SIMCA) and partial least-squares discriminant analysis (PLS-DA) methods. All NIR models were established using raw spectra only. Performance of models was evaluated in terms of number of false positives, number of false negatives, sensitivity (Eq. (1)), specificity (Eq. (2)) and accuracy (Eq. (3)), which is defined as:

$$
\text { Specificity }=\frac{\mathrm{TN}}{\mathrm{TN}+\mathrm{FP}} \times 100
$$

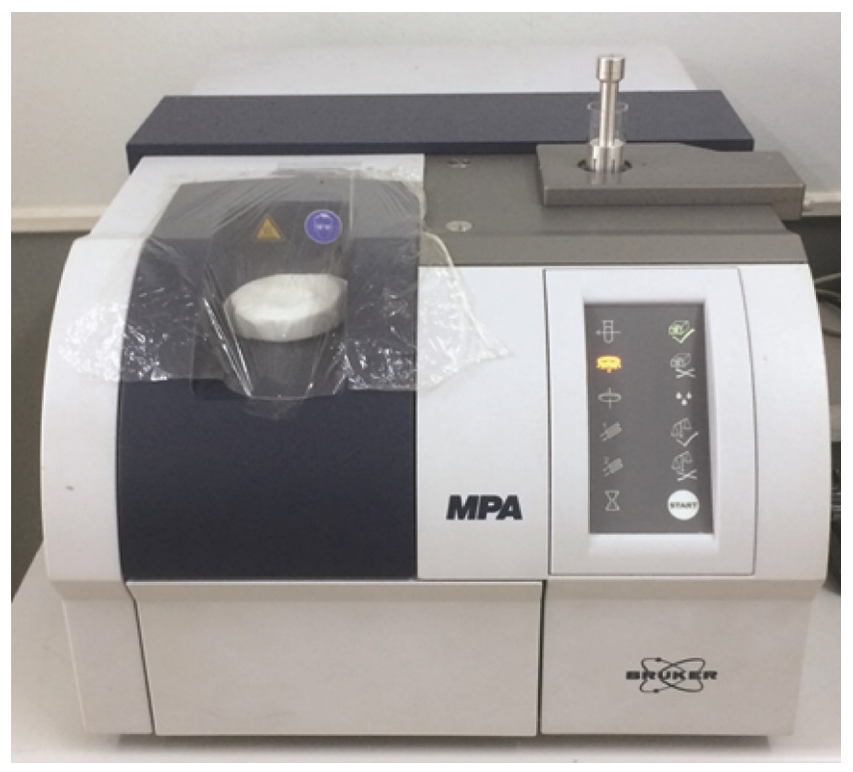

Fig. 1. Syrup spectra measurement by FT-NIR spectrometer.

$$
\begin{gathered}
\text { Sensitivity }=\frac{\mathrm{TP}}{\mathrm{TP}+\mathrm{FN}} \times 100, \\
\text { Accuracy }=\frac{\mathrm{TP}+\mathrm{TN}}{\mathrm{TP}+\mathrm{TN}+\mathrm{FP}+\mathrm{FN}} \times 100,
\end{gathered}
$$

where TP, FN, TN and FP are the numbers of true positives, false negatives, true negatives and false positives, respectively.

\subsection{Overall precision test}

Repeatability and reproducibility were assessed for describing overall precision test. One of the syrup sample (MPS3) was selected to determine repeatability and reproducibility. Repeatability was performed by NIR spectra scanning on selected sample with 10 duplicates under the same conditions. Reproducibility was performed on same selected sample with 10 duplicates under different conditions (lifting and placing). Repeatability and reproducibility are defined as the standard deviation (SD) of absorbance values at $6940 \mathrm{~cm}^{-1}$ (absorbance peaks of sucrose $^{12}$ ) of 10 duplicates.

\section{Results and Discussion}

\subsection{NIR spectra of syrup}

The raw spectra of 30 syrup samples are shown in Fig. 2. The trend and absorbance band of all syrup spectra were similar, except spectrum of MFS1 (maple-flavored syrup). Shapes of raw spectra of all syrup types were no noises. It could be observed by

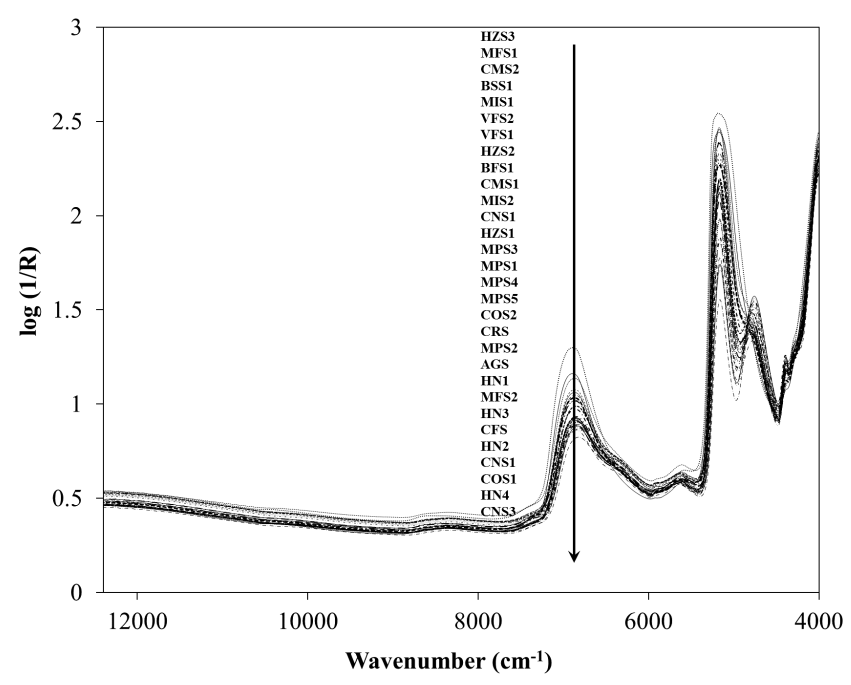

Fig. 2. Raw spectra of syrup. 
naked eyes. However, the spectral data may obtain baseline shrift and/or baseline offset, which led to shift in absorbance peaks. These problems can be resolved using the second derivative method. Figure 3 shows the second derivative spectra of all syrup types. Spectra of all syrup types show obvious peak at $6940 \mathrm{~cm}^{-1}(1440 \mathrm{~nm}), 5900 \mathrm{~cm}^{-1}(1650 \mathrm{~nm})$, $5160 \mathrm{~cm}^{-1} \quad(1940 \mathrm{~nm}), \quad 4810 \mathrm{~cm}^{-1} \quad(2080 \mathrm{~nm})$, $4390 \mathrm{~cm}^{-1}(2276 \mathrm{~nm}), 4300 \mathrm{~cm}^{-1}(2325 \mathrm{~nm})$ and $4000 \mathrm{~cm}^{-1}(2500 \mathrm{~nm})$. The peaks at $6940 \mathrm{~cm}^{-1}$ and $4810 \mathrm{~cm}^{-1}$ correspond to first overtone of the $\mathrm{O}-\mathrm{H}$ stretching and combination of $\mathrm{O}-\mathrm{H}$ stretching and deformation band of sucrose. ${ }^{12}$ Figures 2 and 3 show that the different type of syrup affected the spectral level at $6940 \mathrm{~cm}^{-1}$, which is the absorbance band of sucrose. The prominent peak at $5160 \mathrm{~cm}^{-1}$ is a combination of $\mathrm{O}-\mathrm{H}$ starching and deformation of water. ${ }^{12}$ Absorbance bands of starch reveal at $4390 \mathrm{~cm}^{-1}$ and $4000 \mathrm{~cm}^{-1}$ (i.e., combination of O-H and $\mathrm{C}-\mathrm{C}$ stretching and combination of $\mathrm{C}-\mathrm{H}$ and $\mathrm{C}-\mathrm{C}$ stretching, respectively). ${ }^{12}$

\subsection{NIR identification models}

Figure 4 shows PCA of syrup spectra on factor 1 (PC1) and factor 2 (PC2). The MFS1 was further separated from other syrup types which posited on positive axis of PC1 and the HZS3 was separated from other syrup types on positive axis of PC1 also. Five types of syrup, including HN2, HN3, HN4, CFS and CNS3, were separated from other syrup types on positive axis of PC2. Three types of syrup, which include HN1, AGS and CNS2, were separated from other syrup types on negative axis of PC1. Nine types of syrup that include CMS1, CMS2, HZS2, MIS1, MIS2, VFS1, VFS2, BSS1 and BFS1

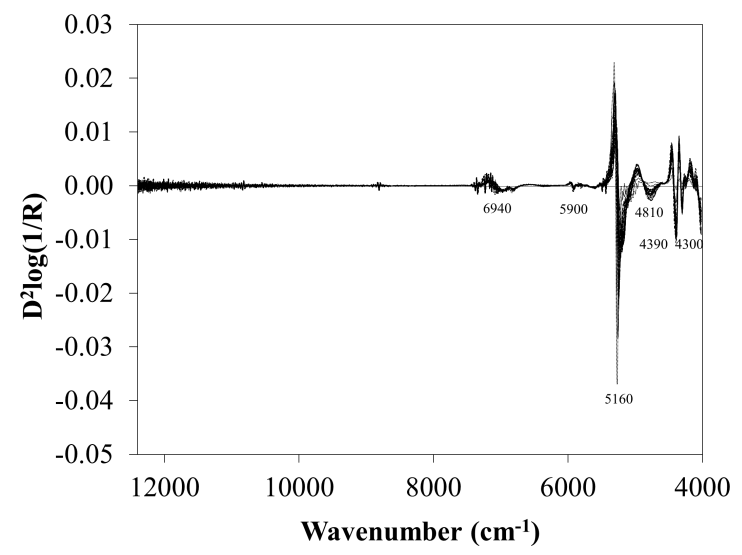

Fig. 3. Second derivative spectra of syrup.

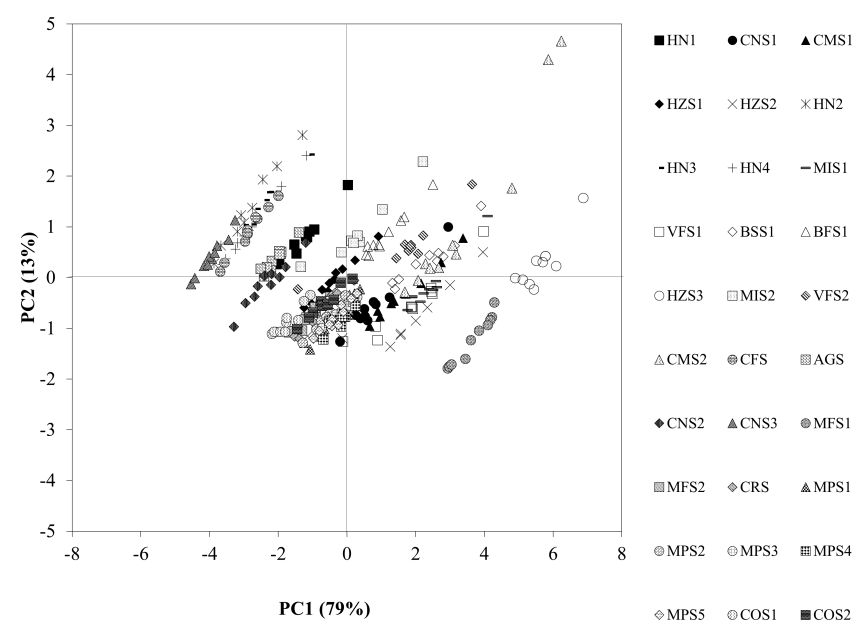

Fig. 4. PCA of syrup spectra.

were distributed on positive axis of $\mathrm{PC} 1$. The large group of syrup consisted of MPS1, MPS2, MPS3, MPS4, MPS5, CRS, MFS2, COS1, COS2, CNS1 and HZS1, clustered around center of PCA plot. Comparing PCA model from FT-NIR with IR spectroscopy for classification of maple ${ }^{1}$ and agave syrup, ${ }^{13}$ we observed that IR spectroscopy could better classify type of syrup than FT-NIR spectroscopy but it needs a sample preparation although simple. ${ }^{14}$

Figure 5 shows $x$-loading plot of PC1 and PC2 from PCA analysis. PC1 and $\mathrm{PC} 2$ presented large positive or negative loading values at $6942 \mathrm{~cm}^{-1}$ $(1440 \mathrm{~nm}), 6329 \mathrm{~cm}^{-1}(1580 \mathrm{~nm}), 5155 \mathrm{~cm}^{-1}(1940 \mathrm{~nm})$ and $4690 \mathrm{~cm}^{-1}(2132 \mathrm{~nm})$, which were important concerned components for separation type of syrup by the PCA method. The peak of loading values at $6942 \mathrm{~cm}^{-1}(1440 \mathrm{~nm})$ and $6329 \mathrm{~cm}^{-1}(1580 \mathrm{~nm})$ corresponded to the $\mathrm{O}-\mathrm{H}$ stretching first overtone of

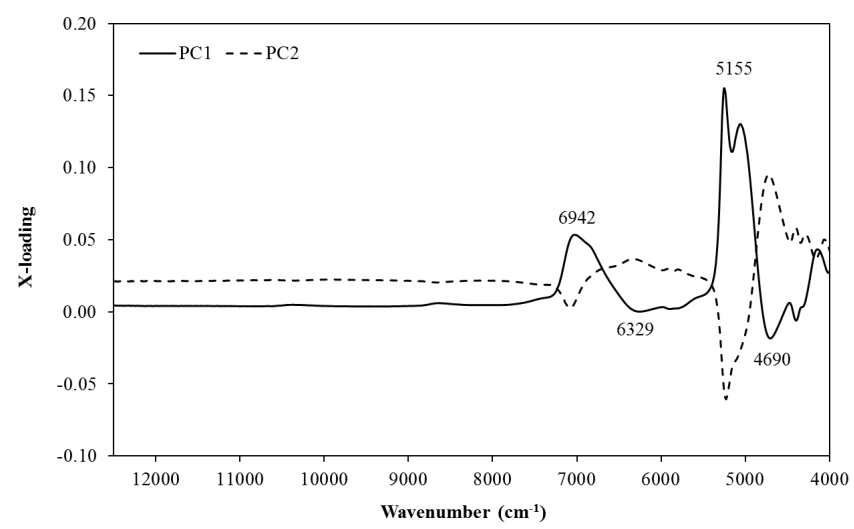

Fig. 5. $x$-loading plot of PC1 and PC2 from PCA analysis. 
sucrose and the $\mathrm{O}-\mathrm{H}$ stretching first overtone of glucose, respectively. ${ }^{12}$ It is clear that different types of syrup could separate by NIR absorbance band of sucrose and glucose, which is the main chemical composition of syrup. Therefore, it is possible for using NIR spectra of syrup to identify syrup type. In addition, the prominent peak at $5155 \mathrm{~cm}^{-1}(1940 \mathrm{~nm})$ was combination absorbance of O-H stretching and deformation of water. ${ }^{12}$

Table 1 presents the result of identification types of syrup using soft independent modeling by SIMCA. Identification models using the SIMCA method showed sensitivity between $33.3 \%$ and $100.0 \%$, whereas specificity of all models was $100 \%$. Most models (AGS, BSS, BFS, CNS1, CNS2, CNS3, CRS1, CFS, COS1, COS2, HZS1, HN1, HN2, MFS1, MFS2, CRS, MIS2, VFS1 and VFS2) presented $100 \%$ of sensitivity, specificity and accuracy. In contrast, some models (CRS2, HZS2, HZS3, HN3, HN4, MPS1, MPS2, MPS3, MPS4, MPS5 and
MIS1) gave percentage of sensitivity of $33.3-83.3 \%$ due to false negative of identification types of syrup obtained on these models, especially all of maple syrup models. This result presented that maple syrup models could not identify among maple syrup with various sources. These consequences were obtained in previous research ${ }^{13}$ studied on classification and discrimination of agave syrup, which reported that NIR spectroscopy was not possible to classify and discriminate among agave syrup with different origins or between syrups from the variant species. This finding indicated that these identification models could be used for self and other types of syrup. The accuracy of identification models using the SIMCA method was more than $93.3 \%$. Therefore, identification models created using SIMCA technique were applicable. The results of identification syrup type using PLS-DA are shown in Table 2. Percentages of sensitivity on identification syrup types by PLS-DA models were

Table 1. Results of identification types of syrup using SIMCA.

\begin{tabular}{lccccc}
\hline Models & False positive & False negative & Specificity $(\%)$ & Sensitivity $(\%)$ & Accuracy $(\%)$ \\
\hline AGS & 0 & 0 & 100.0 & 100.0 & 100.0 \\
BSS & 0 & 0 & 100.0 & 100.0 & 100.0 \\
BFS & 0 & 0 & 100.0 & 100.0 & 100.0 \\
CNS1 & 0 & 0 & 100.0 & 100.0 & 100.0 \\
CNS2 & 0 & 0 & 100.0 & 100.0 & 100.0 \\
CNS3 & 0 & 0 & 100.0 & 100.0 & 100.0 \\
CRS1 & 0 & 0 & 100.0 & 100.0 & 100.0 \\
CRS2 & 0 & 14 & 100.0 & 41.7 & 95.3 \\
CFS & 0 & 0 & 100.0 & 100.0 & 100.0 \\
COS1 & 0 & 0 & 100.0 & 100.0 & 100.0 \\
COS2 & 0 & 0 & 100.0 & 100.0 & 100.0 \\
HZS1 & 0 & 0 & 100.0 & 100.0 & 100.0 \\
HZS2 & 0 & 6 & 100.0 & 62.5 & 98.0 \\
HZS3 & 0 & 18 & 100.0 & 35.7 & 93.9 \\
HN1 & 0 & 0 & 100.0 & 100.0 & 100.0 \\
HN2 & 0 & 0 & 100.0 & 100.0 & 100.0 \\
HN3 & 0 & 2 & 100.0 & 83.3 & 99.3 \\
HN4 & 0 & 2 & 100.0 & 81.8 & 99.3 \\
MFS1 & 0 & 0 & 100.0 & 100.0 & 100.0 \\
MFS2 & 0 & 1 & 100.0 & 100.0 & 100.0 \\
CRS & 0 & 0 & 100.0 & 100.0 & 100.0 \\
MPS1 & 0 & 0 & 100.0 & 33.3 & 93.3 \\
MPS2 & 0 & 100.0 & 83.3 & 99.3 \\
MPS3 & 0 & 0 & 100.0 & 38.5 & 94.6 \\
MPS4 & 0 & 0 & 100.0 & 40.0 & 94.9 \\
MPS5 & 0 & 0 & 100.0 & 58.8 & 97.6 \\
MIS1 & 0 & 0 & 100.0 & 100.0 & 100.0 \\
MIS2 & 0 & 0 & 100.0 & 100.0 & 100.0 \\
VFS1 & 0 & 0 & 100.0 & 0 \\
VFS2 & 0 & 0 & 0.0 & \\
\hline & 0 & 0 & 0.0 & \\
\hline
\end{tabular}


Table 2. Results of identification types of syrup using PLS-DA

\begin{tabular}{|c|c|c|c|c|c|}
\hline Models & False positive & False negative & Specificity (\%) & Sensitivity (\%) & Accuracy (\%) \\
\hline AGS & 0 & 0 & 100.0 & 100.0 & 100.0 \\
\hline $\mathrm{BSS}$ & 10 & 0 & 96.6 & 0.0 & 96.6 \\
\hline BFS & 10 & 0 & 96.6 & 0.0 & 96.6 \\
\hline CNS1 & 10 & 0 & 96.6 & 0.0 & 96.6 \\
\hline CNS2 & 7 & 0 & 97.6 & 100.0 & 97.6 \\
\hline CNS3 & 5 & 0 & 98.3 & 100.0 & 98.3 \\
\hline CRS1 & 10 & 0 & 96.6 & 0.0 & 96.6 \\
\hline CRS2 & 10 & 0 & 96.6 & 0.0 & 96.6 \\
\hline CFS & 10 & 0 & 96.6 & 0.0 & 96.6 \\
\hline COS1 & 10 & 0 & 96.6 & 0.0 & 96.6 \\
\hline COS2 & 10 & 0 & 96.6 & 0.0 & 96.6 \\
\hline HZS1 & 8 & 0 & 97.3 & 100.0 & 97.3 \\
\hline HZS2 & 0 & 0 & 100.0 & 100.0 & 100.0 \\
\hline HZS3 & 10 & 0 & 96.7 & 0.0 & 96.7 \\
\hline HN1 & 10 & 0 & 96.6 & 0.0 & 96.6 \\
\hline $\mathrm{HN} 2$ & 7 & 0 & 97.6 & 100.0 & 97.6 \\
\hline HN3 & 6 & 0 & 98.0 & 100.0 & 98.0 \\
\hline HN4 & 0 & 0 & 100.0 & 100.0 & 100.0 \\
\hline MFS1 & 10 & 0 & 96.6 & 0.0 & 96.6 \\
\hline MFS2 & 0 & 0 & 100.0 & 100.0 & 100.0 \\
\hline CRS & 0 & 0 & 100.0 & 100.0 & 100.0 \\
\hline MPS1 & 10 & 0 & 96.6 & 0.0 & 96.6 \\
\hline MPS2 & 0 & 0 & 100.0 & 100.0 & 100.0 \\
\hline MPS3 & 10 & 0 & 96.6 & 0.0 & 96.6 \\
\hline MPS4 & 10 & 0 & 96.6 & 0.0 & 96.6 \\
\hline MPS5 & 10 & 0 & 96.6 & 0.0 & 96.6 \\
\hline MIS1 & 10 & 0 & 96.6 & 0.0 & 96.6 \\
\hline MIS2 & 8 & 0 & 97.3 & 0.0 & 97.3 \\
\hline VFS1 & 0 & 0 & 100.0 & 0.0 & 100.0 \\
\hline VFS2 & 0 & 0 & 100.0 & 0.0 & 100.0 \\
\hline
\end{tabular}

$0 \%$ and $100 \%$ and specificity of all models was more than $96.6 \%$. The false positive was obtained on most identification models (BSS, BFS, CNS1, CNS2, CNS3, CRS1, CRS2, CFS, COS1, COS2, HZS1, HZS3, HN1, HN2, HN3, MFS1, MPS1, MPS3, MPS4, MPS5, MIS1 and MIS2) using the PLS-DA method, and false negative of these models was 0.0. This finding indicated that these PLS-DA models could be better for identification of other types of syrup but not for self-identification.
However, some models (AGS, HZS2, HN4, MFS2, CRS, MPS2, VFS1 and VFS2) presented that the numbers of false positive and false negative were 0 . The accuracy of all identification models was more than $96.6 \%$. These identification models established by the PLS-DA method were applicable.

Table 3 shows results of discrimination of maple syrup from other syrup types by SIMCA and PLS-DA. Discrimination of maple syrup using the SIMCA method showed accuracy between

Table 3. Results of discrimination maple syrup from other syrup types using SIMCA and PLS-DA.

\begin{tabular}{lccccccc}
\hline & \multicolumn{3}{c}{ SIMCA } & & \multicolumn{3}{c}{ PLS-DA } \\
\cline { 2 - 3 } Syrup type & Sensitivity (\%) & Specificity (\%) & Accuracy (\%) & Sensitivity (\%) & Specificity (\%) & Accuracy (\%) \\
\hline Maple syrup & 100.0 & 99.6 & 99.7 & & 100.0 & 100.0 & 100.0 \\
Other syrup & 83.2 & 0.0 & 83.2 & & 100.0 & 100.0 & 100.0 \\
\hline
\end{tabular}


Table 4. Overall precision test.

\begin{tabular}{cccccc}
\hline & \multicolumn{2}{c}{ Repeatability test } & & Reproducibility test \\
\cline { 2 - 3 } Overall precision test & Mean & SD & & Mean & SD \\
\hline $\begin{array}{c}\text { Absorbance values } \\
\text { at } 6940 \mathrm{~cm}^{-1}\end{array}$ & 0.719 & 0.001 & 0.714 & 0.010 \\
\hline
\end{tabular}

Note: SD: standard deviation.

$83.2 \%$ and $99.7 \%$, whereas the PLS-DA method showed $100 \%$ accuracy. These results indicated that FT-NIR spectroscopy combined with PLS-DA method was high-performance technique for discrimination of maple syrup from other syrups.

\subsection{Overall precision test}

Table 4 shows repeatability and reproducibility at absorbance values at $6940 \mathrm{~cm}^{-1}$. The SD values of repeatability and reproducibility were 0.001 and 0.010 , respectively. The SD of both tests shows the indication of precision of NIR instrument on measurement of syrup spectra under same and different conditions. Repeatability test showed that the SD was $0.14 \%$ of the mean value $(0.719)$ and reproducibility was $1.4 \%$ of the mean value $(0.714)$. This indicated that measurement of syrup spectra using FT-NIR instrument showed excellent precision.

\section{Conclusion}

The performance of syrup type identification and discrimination of maple syrup and other syrup types using FT-NIR spectroscopy were studied in this research. The results showed that FT-NIR combined with SIMCA and PLS-DA has high potential as methods for identification of syrup type and discrimination of maple syrup and other syrups. The FT-NIR spectroscopy in combination with SIMCA and PLS-DA can therefore be used as a fast, lost cost, simple and nondestructive tool for identification and discrimination of syrup. However, the FT-NIR spectroscopy with SIMCA and PLS-DA could not be applied to identify maple syrup among various sources. Nevertheless, FTNIR models that developed from maple syrup using SIMCA and PLS-DA presented excellent ability for discrimination of maple syrup from other syrup types.

\section{Acknowledgments}

The authors gratefully acknowledge the instrument, fund and software support provided by the NIRS Research Center for Agricultural Product and Food, Curriculum of Agricultural Engineering, Department of Mechanical Engineering, Faculty of Engineering, King Mongkut's Institute of Technology Ladkrabang, Bangkok, Thailand. The opinions expressed in this research paper are those of the authors alone.

\section{References}

1. E. Mellado-Mojica, N. P. Seeram, M. G. López, "Comparative analysis of maple syrups and natural sweeteners: Carbohydrates composition and classification (differentiation) by HPAEC-PAD and FTIR spectroscopy-chemometrics," J. Food Compos. Anal. 52, 1-8 (2016).

2. J. G. Stuckel, N. H. Low, "Maple syrup authenticity analysis by anion-exchange liquid chromatography with pulsed amperometric detection," J. Agric. Food Chem. 43, 3046-3051 (1995).

3. J. G. Stuckel, N. H. Low, "The chemical composition of 80 pure maple syrup samples produced in North America," Food Res. Int. J. 29, 313-319 (1996).

4. T. D. Perkins, A. K. Van den Berg, "Maple syrup Production, composition, chemistry, and sensory characteristics," Adv. Food Nutn Res. 56, 101-143 (2009).

5. L. Luqing, W. Lingdong, N. Jingming, Z. Zhengzhu, "Detection and quantification of sugar and glucose syrup in roasted green tea using near infrared spectroscopy," J. Near Infrared Spectrosc. 23, 317$325(2015)$.

6. J. D. Kelly, C. Petisco, G. Downey, "Potential of near infrared transflectance spectroscopy to detect adulteration of Irish honey by beet invert syrup and high fructose corn syrup," J. Near Infrared Spectrosc. 14, 139-146 (2006).

7. S. Li, X. Zhang, Y. Shan, D. Su, Q. Ma, R. Wen, J. Li, "Qualitative and quantitative detection of honey adulterated with high-fructose corn syrup and maltose syrup by using near-infrared," Food Chem. 218, 231-236 (2017).

8. P. Veleva-Doneva, T. Draganova, S. Atanassova, R. Tsenkova, "Detection of bacterial contamination in milk using NIR spectroscopy and two classification methods - SIMCA and neuro-fuzzy classifier," 3rd IFAC Processing, Vol. 43, pp. 225-229 (2010).

9. S. Serranti, D. Cesare, F. Marini, G. Bonifazi, "Classification of oat and groat kernels using NIR 
hyperspectral imaging," Talanta 103, 276-284 (2013).

10. B. Jamshidi, E. Mohajerani, J. Jamshidi, "Developing a Vis/NIR spectroscopic system for fast and non-destructive pesticide residue monitoring in agricultural product," Measurement 89, 1-6 (2016).

11. A. López-Maestresalas, A. C. Keresztes, M. Goodarzi, S. Arazuri, C. Jarén, W. Saeys, "Nondestructive detection of blackspot in potatoes by Vis-NIR and SWIR hyperspectral imaging," Food Control 70, 229-241 (2016).
12. B. G. Osborne, T. Fearn, Near Infrared Spectroscopy in Food Analysis, 227pp., Longman Science \& Technical, UK (1986).

13. E. Mellado-Mojica, M. G. López, "Identification, classification, and discrimination of agave syrups from natural sweeteners by infrared spectroscopy and HPAEC-PAD," Food Chem. 167, 349-357 (2015).

14. C. Alamprese, E. Casiraghi, "Application of FT-NIR and FT-IR spectroscopy to fish fillet authentication," LWT-Food Sci. Technol. 63(1), $720-725$ (2015). 